\title{
PPM-Extended (PPMX) - a catalogue of positions and proper motions ${ }^{\star}$
}

\author{
S. Röser ${ }^{1}$, E. Schilbach ${ }^{1}$, H. Schwan ${ }^{1}$, N. V. Kharchenko ${ }^{1,2,3}$, A. E. Piskunov ${ }^{1,3,4}$, and R.-D. Scholz ${ }^{3}$ \\ 1 Astronomisches Rechen-Institut, Zentrum für Astronomie der Universität Heidelberg, Mönchhofstraße 12-14, \\ 69120 Heidelberg, Germany \\ e-mail: [roeser; elena;schwan;nkhar;apiskunov]@ari.uni-heidelberg.de \\ 2 Main Astronomical Observatory, 27 Academica Zabolotnogo Str., 03680 Kiev, Ukraine \\ e-mail: nkhar@mao.kiev.ua \\ 3 Astrophysikalisches Institut Potsdam, An der Sternwarte 16, 14482 Potsdam, Germany \\ e-mail: [nkharchenko; apiskunov;rdscholz]@aip.de \\ ${ }^{4}$ Institute of Astronomy of the Russian Acad. Sci., 48 Pyatnitskaya Str., 109017 Moscow, Russia \\ e-mail: piskunov@inasan.rssi.ru
}

Received 13 March 2008 / Accepted 9 May 2008

\section{ABSTRACT}

\begin{abstract}
Aims. We build a catalogue PPM-Extended (PPMX) on the ICRS system which is complete down to a well-defined limiting magnitude and contains the best presently available proper motions suited for kinematic studies in the Galaxy.

Methods. We perform a rigorous weighted least-squares adjustment of individual observations, spread over more than a century, to determine mean positions and proper motions. The stellar content of PPMX is taken from GSC 1.2 supplemented by catalogues like ARIHIP, PPM and Tycho-2 at the bright end. All observations have been weighted according to their individual accuracy. The catalogue has been screened towards rejecting false entries in the various source catalogues.

Results. PPM-Extended (PPMX) is a catalogue of 18088919 stars containing astrometric and photometric information. Its limiting magnitude is about 15.2 in the GSC photometric system. PPMX consists of three parts: a) a survey complete down to $R_{\mathrm{U}}=12.8$ in the magnitude system of UCAC2; b) additional stars of high-precision proper motions, and c) all other stars from GSC 1.2 identified in 2MASS. The typical accuracy of the proper motions is 2 mas/y for 66 percent of the survey stars (a) and the high-precision stars (b), and about 10 mas/y for all other stars. PPMX contains photometric information from ASCC-2.5 and 2MASS.
\end{abstract}

Key words. catalogs - astrometry

\section{Introduction}

According to IAU Resolution B2 of the XXIIIrd General Assembly (1997), the Hipparcos Catalogue (ESA 1997) is the primary realisation of the International Celestial Reference System (ICRS) at optical wavelengths. Since then, it has been the aim of the astrometric community to extend the Hipparcos system to higher star densities and fainter limiting magnitudes. The first and most important catalogue is Tycho-2 (Høg et al. 2000b), based on observations of the Tycho experiment onboard the ESA-Hipparcos satellite. The old-epoch observations of Tycho-2 were taken from new reductions (Urban et al. 1998) of the observations of the Astrographic Catalogue. Tycho-2 contains about 2.5 million stars and is 90 percent complete down to $V=11.5$. The Tycho $B$ and $V$ magnitudes were transferred to the Johnson system in ASCC-2.5 (Kharchenko 2001).

In 2004, the UCAC2 catalogue (Zacharias et al. 2004) was published. It is based on a new full-sky survey made with a newly developed astrographic camera from 1998 to 2004. The first epochs are taken from yet unpublished remeasures of the AGK2 plates and scans from the NPM and SPM plates, also known as the Yellow Sky (YS). To a minor extent, old epochs

\footnotetext{
* Star catalog is only available in electronic form at the CDS via anonymous ftp to cdsarc.u-strasbg.fr (130.79.128.5) or via http://cdsweb.u-strasbg.fr/cgi-bin/qcat?J/A+A/488/401
}

come also from AC and smaller astrometric catalogues of the last century. UCAC2 is not yet complete; the declination zone from about +40 deg to the north pole is still missing. UCAC2 contains some 48 million stars down to $R_{\mathrm{U}}=16 \mathrm{mag}$. Proper motion errors are about 1-3 mas/yr for stars to 12th mag, and about 4-7 mas/yr for fainter stars to 16 th mag.

The largest catalogue in the optical regime is USNO B1.0 (Monet et al. 2003) with more than one billion objects. However, USNO B1.0 is not in the system of ICRS; it contains relative, not absolute proper motions (see Monet et al. 2003). A comparison of USNO B1.0 and UCAC2 performed in the present work yielded systematic differences (in areas of square degrees) of up to $5 \mathrm{mas} / \mathrm{y}$ in proper motion and up to $0.5 \mathrm{arcsec}$ at present epoch.

For kinematical studies in the Milky Way, more precisely in the closer neighbourhood of the Sun, a catalogue of proper motions in the ICRS system and with a well-defined completeness limit is indispensable. At Astronomisches RechenInstitut, Heidelberg, we therefore started an initiative to extend the Tycho-2 system to fainter magnitudes using all the relevant astrometric observations of the last century, and perform a rigorous least-squares adjustment to derive proper motions, instead of determining proper motions from the diffences of positions from two epochs. 
The first result of this effort is PPM-Extended (PPMX). This paper summarizes the astrometric and photometric sources from which PPMX is build together with its properties. The aim of having a proper motion catalogue of homogeneous accuracy and complete down to a well-defined limiting magnitude in the visual regime (considerably fainter than Tycho-2) is not achievable at present. The main reason for this is the inhomogeneity of oldepoch observations, mainly of AC. So, PPMX is a compromise between having a bright completeness limit and proper motions of homogeneous accuracy and a fainter completeness limit with a more inhomogeneous distribution of the accuracies of proper motions.

In the next section we summarise the properties of the source catalogues used in PPMX, then we briefly describe the construction of the catalogue, and finish with an overview of the properties of PPMX.

\section{Observational catalogues}

a) The Astrographic Catalogue

The Astrographic Catalogue project (initiated in 1887) was one of the largest enterprises ever undertaken in astronomy. It resulted in about 10 million measurements of rectangular plate coordinates for some 4.6 million different stars. AC is a double coverage of the whole sky. The observations and plate measurements were carried out by 20 observatories all over the world. Each observatory was responsible for a declination zone on the sky. The printed coordinates have been independently digitised at the CDS, Strasbourg (partially), at the US Naval Observatory and the Sternberg Institute in Moscow. For PPMX we made use of information contained in each of the three data sets. A full reduction of the AC in the Hipparcos system is given in Urban et al. (2001), called AC2000.2, which presents averages of the individual measurements per star. We have attributed weights for the individual positions in the AC zones according to the accuracy of the position measurements in the sources: these weights were taken from Röser \& Høg (1993).

b) The Guide Star Catalog

The Guide Star Catalog (GSC 1.0, Lasker et al. 1990) was constructed to support pointing and target acquisition for the Hubble Space Telescope (HST). For the construction of PPMX we used the GSC 1.2 (Morrison et al. 2001), which saw a considerable improvement of the astrometric quality due to a reduction of systematic errors as functions of the stellar locus on the plate and on the stellar magnitude. Typical mean errors of a coordinate in GSC 1.2 are about 0.3 arcsec (Morrison et al. 2001). GSC 1.2 contains some 25 million observations for about 18 million individual stars. As GSC 1.2 is not on the ICRS system (represented by Tycho-2), it was reduced to it via the procedure described in Sect. 3.3.

c) 2MASS

The Two Micron All Sky Survey (Skrutskie et al. 2006), 2MASS, is a complete Sky-Survey in the $J, H$ and $K_{\mathrm{s}}$ bands performed in the years from 1997 to 2001. 2MASS is also a source of accurate astrometric positions. Its astrometric accuracy is 100 mas $(1 \sigma)$ relative to the Hipparcos reference frame for $K_{\mathrm{s}}<14$ (Skrutskie et al. 2006). For the construction of PPMX we used the Point Source Catalog of some 471 million entries. d) Tycho-2

The Tycho-2 catalogue (Høg et al. 2000b) is a compiled astrometric catalogue containing positions and proper motions as well as two-colour photometric data for the 2.5 million brightest stars in the sky. The modern-epoch $(\approx 1991.25)$ positions and magnitudes were obtained from the Tycho star mapper observations onboard the Hipparcos satellite. The mean standard errors in astrometry range from about 4 mas at $V<7.0$ mag to about 90 mas for stars fainter than $V=12.0$ mag. From Tycho-2 we exclusively used the 1991.25-epoch positions measured with the Hipparcos star mapper.

\section{e) $\mathrm{CMC} 14$}

CMC14 (Copenhagen Univ. Obs. et al. 2006) is the latest catalogue from the Carlsberg Meridian Telescope, observed in the years 1999 to the end of 2005. The data are obtained with a $2 k \times 2 k$ CCD camera operating in a drift scan mode. Its magnitude limit is 17 (Sloan r') and the positional accuracy is from 35 to 100 mas. The observations cover the declination band from -30 to $+50 \mathrm{deg}$, and the catalogue contains some 95 million stars.

f) The Bordeaux Carte-du-Ciel (CdC2000) catalogue

The Carte-du-Ciel project accompanied the Astrographic Catalogue. Each observatory was supposed to map its zone in a single overlap with 3 exposures per plate, going 2 to 3 mag fainter than AC. This project was never completed. The CdC2000 (Rapaport et al. 2006) catalogue provides astrometric positions of the stars present on 512 Carte du Ciel plates archived at Bordeaux Observatory covering a declination range $+11^{\circ} \leq \delta \leq+18^{\circ}$. It contains the positions of 344781 stars down to $m_{\mathrm{pg}}=17$. The mean positional accuracy is $0.10-0.12^{\prime \prime}$ with mean epoch 1914.7 . This catalogue is particularly valuable for PPMX, because the limiting magnitude of the $\mathrm{AC}$ catalogue in this declination zone is too bright.

g) UCAC2

The second US Naval Observatory CCD Astrograph Catalog, UCAC2, (Zacharias et al. 2004) contains positions and proper motions for some 48 million objects (mostly stars) and covers the sky area from $-90^{\circ}$ to $+40^{\circ}$ in declination, going up to $+52^{\circ}$ in some areas. The accuracy of the positions obtained with the CCD Astrograph in the years 1998 to 2004 is $15-70$ mas, depending on magnitude, with estimated systematic errors of 10 mas or below. UCAC2 is a compiled catalog of positions and proper motions referred to a standard epoch (J2000.0); the original observations are not yet published. In this work we used UCAC2 with its given astrometric position at the catalogue epoch J2000.0.

\section{h) ASCC-2.5}

ASCC-2.5 (Kharchenko 2001) is a catalogue of 2.5 million stars with proper motions in the Hipparcos system. It is a compiled catalogue from sources like Tycho-2 - which overwhelmingly contributes - PPM, Hipparcos and CMC 11 . No astrometric data from ASCC-2.5 entered PPMX; only the $B$ and $V$ magnitudes in the Johnson photometric system were taken instead of the $B_{\mathrm{T}}$ and $V_{\mathrm{T}}$ from Tycho- 2 .

i) PPM

The PPM catalogue (Röser \& Bastian 1991) is a catalogue of positions and proper motions of 378910 stars. It contains the majority of all ground-based astrometric measurements (pre-Hipparcos) from the last century with stars from the AC as first epochs. A rigorous weighted LSQ adjustment was applied to determine positions and motions. In Sect. 2.1 we outline how to incorporate the old PPM into the least-squares 
adjustment for PPMX, and in Sect. 3.3 we describe the reduction of the PPM, which is on the FK5 system, to the ICRS system.

j) ARIHIP

The ARIHIP Catalogue (Wielen et al. 2001) is a combination of the results of the HIPPARCOS astrometry satellite with ground-based data, and contains 90842 stars in total. The typical mean error of an ARIHIP proper motion in the single-star mode (see Wielen et al. 1999) is 0.89 mas/year. In the construction of PPMX, no attempt has been made to improve the astrometric information contained in ARIHIP. Therefore the astrometric data from ARIHIP are simply copied for the stars present in PPMX. Note that the more accurate new reduction of the measurements of the Hipparcos satellite (van Leeuwen 2007) is not used in ARIHIP; also, they arrived too late to be incorporated in PPMX.

k) STARNET

The STARNET catalogue (Röser 1996) has been constructed from GSC 1.0 and a new reduction of the Astrographic Catalogue based on the keypunched dataset from the Sternberg Institute in Moscow. STARNET contains some 4.3 million stars with accuracy of proper motions of 5 mas/y. Unfortunately, the original AC plate coordinates have not been published. As STARNET is not on the ICRS system (represented by Tycho-2), it was reduced to it via the procedure described in Sect. 3.3.

The simple LSQ adjustment described in Sect. 3.4 received positions as input. So, special care has been taken that positions implicitly contained in some of the compilation catalogues from above did not enter the final catalogue more than once. If, for instance, STARNET was used as a source catalogue, AC and GSC 1.2 positions were reconstructed as described in Sect. 2.1. In this case, no positions from AC2000.2 or from the published GSC 1.2 were used. This stresses the importance of publishing original astrometric measurements, and not only derived and/or averaged coordinates. This is especially important in the case of $\mathrm{AC}$, where the full data sets of $x, y$-coordinates reside at USNO and at the Sternberg Institute in Moscow. Because of their enormous importance for proper motions these data sets must be stored in astronomical data centres such as the CDS. The same holds for UCAC2, which is a compiled catalogue, but the original observations from the USNO CCD-astrograph, as well as the positions of the Yellow Sky, have not been published anywhere.

\subsection{Re-construction of the old epochs from PPM and STARNET}

Both catalogues (PPM and STARNET) publish the full covariance matrix of the astrometric parameters (i.e. mean epochs and mean errors of positions and proper motions). In this case we applied a method described by Kopff et al. (1964) to equivalently replace the positions and motions by two normal observations. Let $\bar{T}$ be the mean epoch, $\sigma_{\mathrm{p}}$ and $\sigma_{\mathrm{p} \text {.m. }}$ the mean errors of position and proper motion per coordinate, respectively. Then $w=\sigma_{\mathrm{p}}^{-2}$ and $w_{\mathrm{p} . \mathrm{m} .}=\sigma_{\mathrm{p} . \mathrm{m} .}^{-2}$ are the corresponding weights. Both epochs of normal observations $T_{1}$ and $T_{2}$ and the corresponding weights $w_{1}$ and $w_{2}$ are given from the equations

$$
w=\sum_{i=1, n} w_{i}, w \bar{T}=\sum_{i=1, n} w_{i} T_{i}, w_{\mathrm{p} . \mathrm{m} .}=\sum_{i=1, n} w_{i}\left(T_{i}-\bar{T}\right)^{2}
$$

with $n=2$. Suppose that one of the epochs, e.g. $T_{2}$ is given (known), then all the other quantities are readily determined from the Eq. (1). The positions at the epochs $T_{1}$ and $T_{2}$ are calculated straightforwardly from the positions at epoch 2000.0 and the proper motions.

In the case of a star in STARNET we know that $T_{2}$ is given by its GSC epoch, which is published, and in the case of PPM the second epochs are either AGK3, CMC 1 to 4, or FOCAT-S (Bystrov et al. 1994) on the southern hemisphere. In all cases the normal observations are easily derived, and so we can profit from all the old catalogues used in the construction of PPM. The normal observations have been combined with the new positions from Tycho-2, UCAC2, CMC14 and 2MASS in the LSQ adjustment described in the next section.

\section{Construction of PPMX}

Four major steps were necessary to complete PPMX: a) determining the star-list, b) cross-identifications of the source catalogues with the star-list, c) reducing the individual catalogues to the system of Tycho-2, and finally d) individual LSQ adjustment.

\subsection{The star-list of PPMX}

The star-list of PPMX is a preliminary catalogue with a mean position and a proper motion for each star. It is constructed in the following way: GSC 1.2 was taken as the starting point. It was cross-matched with itself to find stars instead of observations. The cross-matching was on a $15 \times 15$ arcsec window and gave the results discussed in Sect. 3.5. All matches within 3 arcsec were attributed to an individual star. These individual GSC 1.2 stars have been cross-matched with 2 MASS in a $15 \times 15$ arcsec window. GSC 1.2 stars that could not be matched were not included in the star list. If a single star in GSC 1.2 was matched with a single star in 2MASS, this was rated a valid identification. This allows us to find stars with proper motions as large as $0.75 \mathrm{arcsec} / \mathrm{y}$ per coordinate for a typical epoch difference of 20 years between GSC 1.2 and 2MASS, with all the risk of finding spurious proper motions. If more than one match occured within the window, all GSC and 2MASS entries were gathered together and the GSC star pattern was shifted to match the 2MASS pattern. For all the successful matches an average position at 2000.0 and a proper motion was calculated, and this was called star-list 1 . Next, STARNET was cross-matched with star-list 1 in a $3 \times 3$ arcsec window at 2000.0. All STARNET stars that could NOT be identified in star-list 1 were added to it to create star-list 2. The same procedure was adopted for PPM and Tycho-2 to create star-list 3. Finally, AC2000.2 was matched with star-list 3 in a $3 \times 3$ arcsec window at the AC2000.2 epoch. All AC2000.2 stars that could NOT be identified were then cross-matched with 2 MASS in a $3 \times 3$ arcsec window, and those stars with matches were added to build the final star-list.

\subsection{Cross-identifications}

All observational catalogues from Sect. 2 with the exception of ARIHIP were then cross-matched with the star list in a $3 \times$ 3 arcsec window around the star list position at the epoch of the observation. If more than one match occured in this small window, the nearest neighbour was chosen. ARIHIP was only cross-matched after the LSQ adjustment for each star, and the astrometric information was over-written. 


\subsection{Systematic differences to Tycho-2}

All observational catalogues have been cross-matched with Tycho-2 and individual differences in right ascension and declination have been calculated. The individual differences were averaged in bins of $0.5 \times 0.5$ degrees in right ascension and declination, and the systematic differences in each bin determined via a $3 \times 3$ bin moving average filter. This gives an effective area of 2.25 square degrees, which, on average, contains 120 Tycho-2 stars. Only in the case of PPM was a bin size of one degree taken because of the low spatial density of PPM. After subtracting the so-determined spatial systematic differences, magnitudedependent differences within the magnitude range of Tycho-2 were determined and corrected for.

\subsection{The weigthed LSQ adjustment}

Weights $w_{i}$ are attributed to all $\mathrm{n}$ observations of a star according to Sect. 2. Then the resulting covariance matrix of the unknowns (mean position, proper motion) per coordinate is given by Eq. (1). For the mean positions $\bar{x}$ and proper motions $\mu$ the following formulae hold

$\bar{x}=\frac{\sum_{i=1, n} w_{i} x_{i}}{\sum_{i=1, n} w_{i}}, \mu=\frac{\sum_{i=1, n} w_{i} x_{i}\left(T_{i}-\bar{T}\right)}{\sum_{i=1, n} w_{i}\left(T_{i}-\bar{T}\right)^{2}}$.

Automatic tests for unduly large scatter among the measurements (based on the $\chi^{2}$ sum) and automatic elimination of obvious outliers (based on appropriately normalised individual residues) were implemented. All stars having $\chi^{2}$ sums beyond a certain significance limit, but still not showing obvious outliers, were marked as "problem cases" and were given a "P" flag in the catalogue.

No attempt has been made to improve the astrometric quality of stars in ARIHIP; in which case the data were copied from Wielen et al. (2001).

\subsection{Autocorrelation functions}

The plates that form the basis for GSC 1.2 partially overlap, which may result in up to 4 observations per star in this catalogue. We did not only rely on the cross-matching in GSC 1.2, i.e. using GSC 1.2 names, but performed a new one based on coordinates only (described in Sect. 3.1). A by-product of the large self-identification window is the detection of possibly spurious entries in a catalogue. GSC 1.2 is based on Schmidt plates and an automatic object detection was carried out. Spikes around brighter stars on Schmidt plates may give rise to artefacts. The result of the self-identification of GSC 1.2 is the twodimensional two-point autocorrelation function of GSC 1.2 on a $30 \times 30$ arcsec scale which is shown in Figs. 1 and 2, for the northern, and the southern part of the sky, respectively. On a sphere randomly covered with single stars (one entry per star) the two-dimensional autocorrelation function should be completely flat outside the origin. A real excess on top of this flat distribution has to be attributed to physical double stars, which would result in an increase that is radially symmetric around the origin, given that the angular resolution of a catalogue is better than the separation of the binary. Any deviation from radial symmetry has to be considered an artefact.

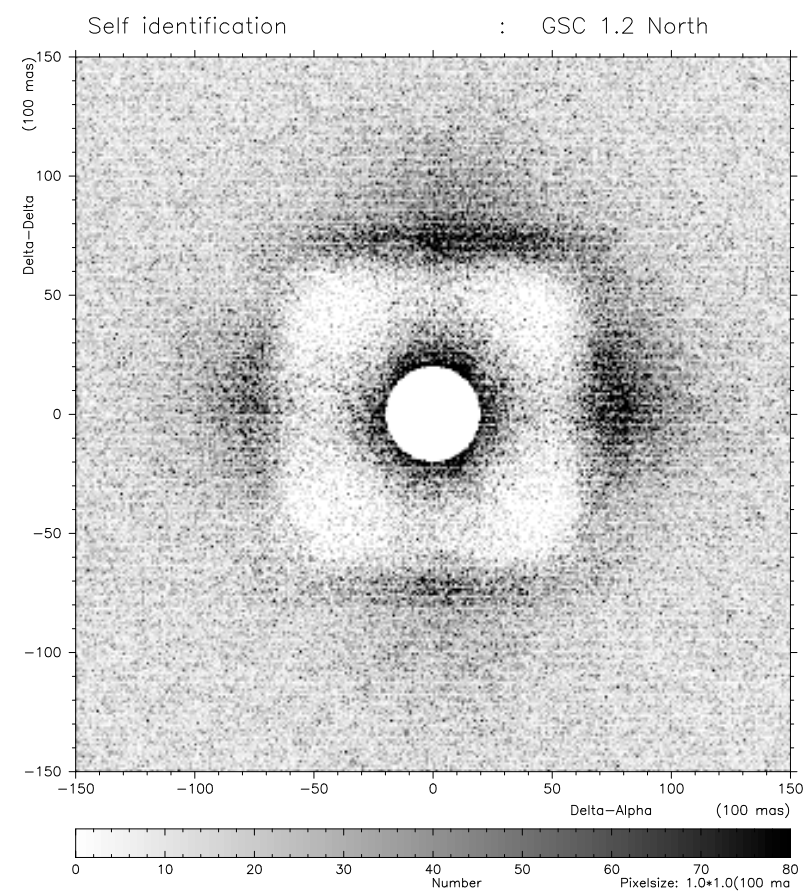

Fig. 1. The two-point autocorrelation function of the northern part of GSC 1.2. The differences in right ascension and declination are given in units of 100 mas. The pixel size is $(100 \mathrm{mas})^{2}$. For further explanation see text.

Self identification

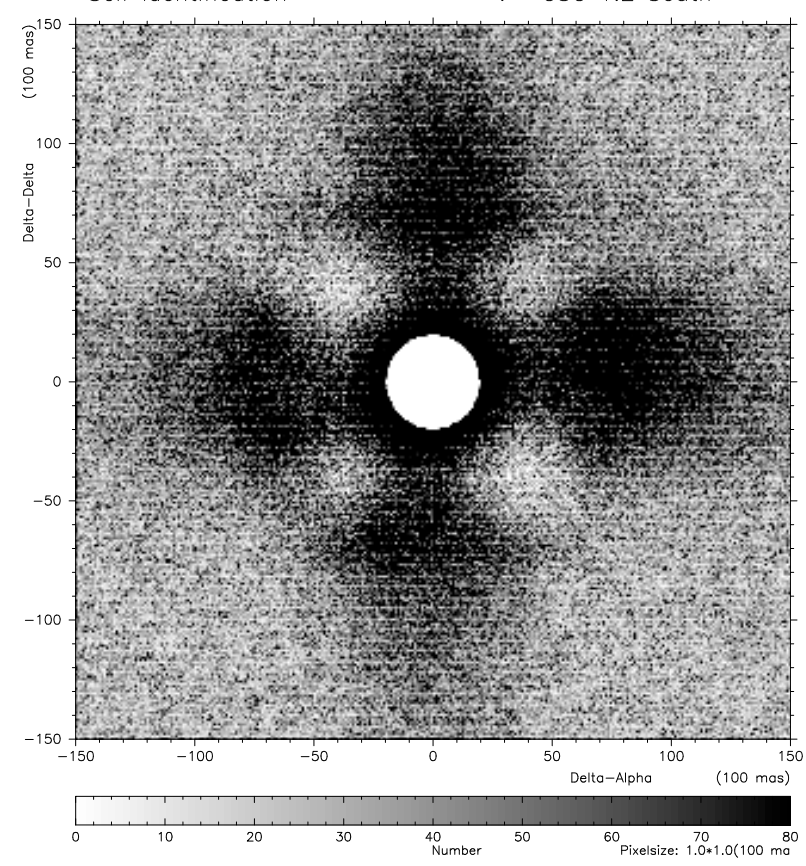

Fig. 2. The same as Fig. 1, but for the southern part of GSC 1.2.

In Figs. 1 and 2, cross-matches inside a radius of 2 arcsec are not plotted, thus generating an empty hole. If a match from two different plates occured within 3 arcsec, this match was attributed to the same star. Separations of less than 3 arcsec cannot be resolved in GSC. One can see the wings of this distribution in both plots. We note that on the northern part based on the Palomar Quick-V survey the authors of GSC 1.2 had already cleaned the catalogue within a central square of $12 \times$ 12 arcsec. However, we still find hints of artefacts from spikes 


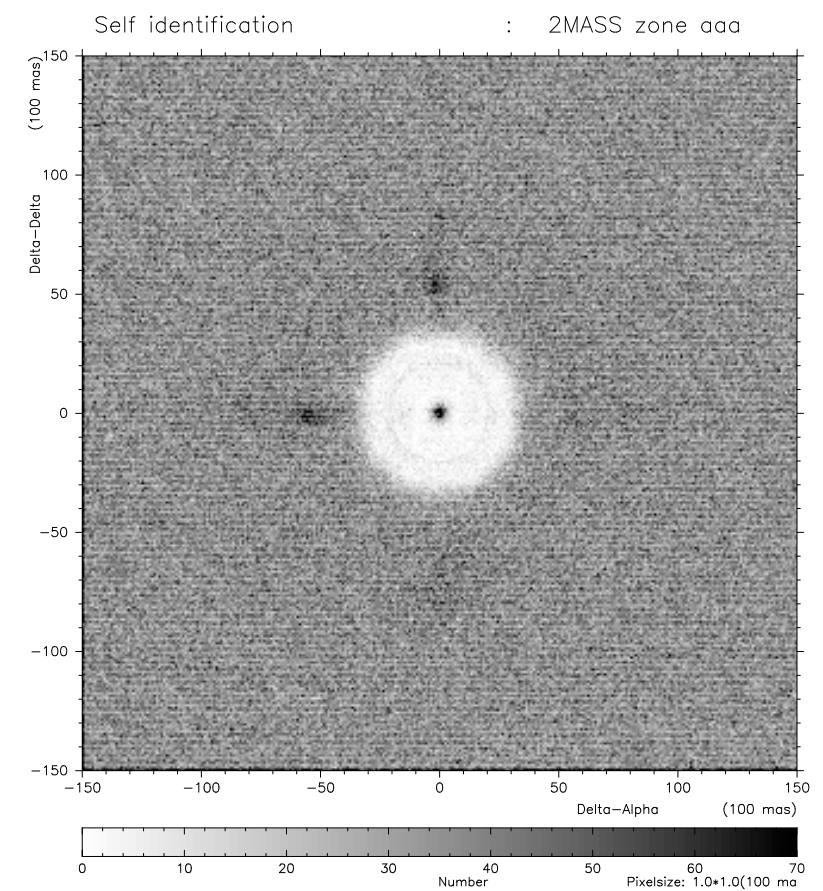

Fig. 3. The autocorrelation function of 2MASS. Here, it is shown only for the 5 million stars on the south polar cap (zone aaa). A full crossmatching of the 0.5 billion stars in 2MASS has not been performed.

on the Schmidt plates. This is more pronounced in the southern part, which is based on deeper plates, and where a different cleaning procedure could have been chosen.

For 2MASS a full autocorrelation of its about half a billion entries has not been performed, only of the subset of 5 million stars from the south polar cap (zone aaa). We assume that this zone is representitative for 2MASS. The result of the autocorrelation of the subset is shown in Fig. 3. If this is representative then 2MASS did an excellent job. The distribution is completely flat outside a radius of 3 arcsec separation, except for a slight indication of spikes north and east of the origin. In these comparisons we did not consider flags in 2MASS. Note that the spikes are mostly inside a radius of 6 arcsec, and the note for the "prox" flag in 2MASS hints that caution is needed if two entries have a distance of less than 6 arcsec. The spikes are more pronounced if the central star is brighter than $K_{\mathrm{S}}<11$, and are practically absent if $K_{\mathrm{s}}>12$.

\section{Properties of PPMX}

The complete catalogue PPMX contains 18088919 stars. For convenience, we sub-divided the catalogue into three disjunct subsets: the survey, additional high-precision stars and all others.

\subsection{Selection of the different subsets}

PPMX uses sky surveys taken in different wavelength bands. The northern part of GSC 1.2, for instance, is observed in the $V$-band, 2MASS in the infrared, and AC in the blue photographic range. Moreover, the different AC zones have different limiting magnitudes mostly due to the efforts invested in manually measuring the stars, which prevents us from simply taking the $\mathrm{AC}$ subset as a magnitude limited sample. In addition, the photometric accuracy in $\mathrm{AC}$ is low and inhomogeneous. Also, the

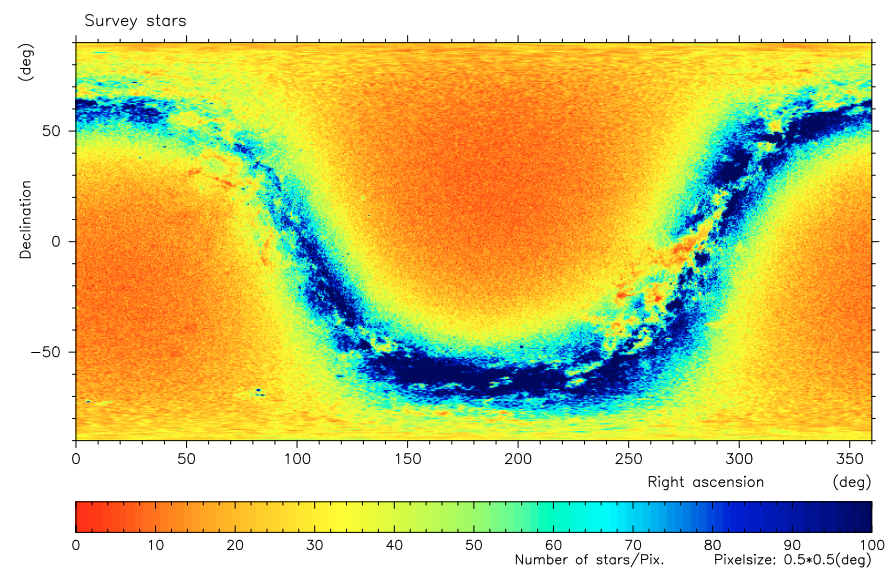

Fig. 4. Number density of stars in the PPMX survey in equatorial coordinates. The bin size is (formally) 0.25 square degrees. No artefacts due to the lay-outs of the photographic surveys are seen here.

photometric accuracy of GSC 1.2 is only about 0.3 mag (Morrison et al. 2001), which would imply a rather soft boundary. Neither can we simply adopt an infrared band from 2MASS, because this would lead to a low completeness limit in the visual in order to take account of very cool or reddened stars, mainly at low galactic latitudes. Piskunov et al. (2008) have introduced an artificial $R_{J}$ band constructed from the $J$ and $H$ magnitudes in 2MASS. This $R_{J}$-band was calibrated towards the observed UCAC2 $R_{U}$ magnitudes. We have taken the $R_{J}$-magnitude of 12.8 as the limiting magnitude of our subset called "survey". This selection was guided by the requirement that the majority of stars in the survey should be stars with observations in the AC to exploit their high accuracy of the proper motions. Altogether there are 5620114 stars in the survey, and they are flagged " $\mathrm{S}$ " in field 26 of the catalogue. Among the survey stars, 3.7 million (66\%) have observations in AC, and therefore highly accurate proper motions, whereas the remaining 1.9 million have typical mean errors of the proper motions of about 10 mas/y (see Fig. 8).

Figure 4 shows the distribution of the survey stars on the sky plotted against right ascension and declination. This presentation has been selected to show that there are no artificial enhancements or gaps related to the different zones or plate borders of the source catalogues.

Another 874934 stars fainter than the survey limit have measurements in the AC and therefore highly accurate proper motions ( $\sigma_{\text {p.m. }}$ peaking at about 3 mas/y per coordinate). They are gathered as "additional high-precision stars" flagged " $H$ " (see Fig. 8). All other stars (11593 871) get the flag "O"; their earliest observation epoch is from GSC 1.2.

For 57334 stars in PPMX the determination of the $R_{J^{-}}$ magnitude was not possible because the magnitudes from 2MASS are contradictory. They are not included in the survey even if they may be bright enough to be members. Instead, they are flagged "H" or "O" in Col. 26 according to the mean error of proper motions.

Users of PPMX can set selection criteria other than the ones we used to define our "survey".

\subsection{The autocorrelation function of PPMX}

Cross-identification of GSC 1.2 with 2MASS solved the majority of the problems with false entries in both catalogues shown in Figs. 1-3; an additional cross-identification with CMC14 in the region between -30 and +50 degrees declination made further 


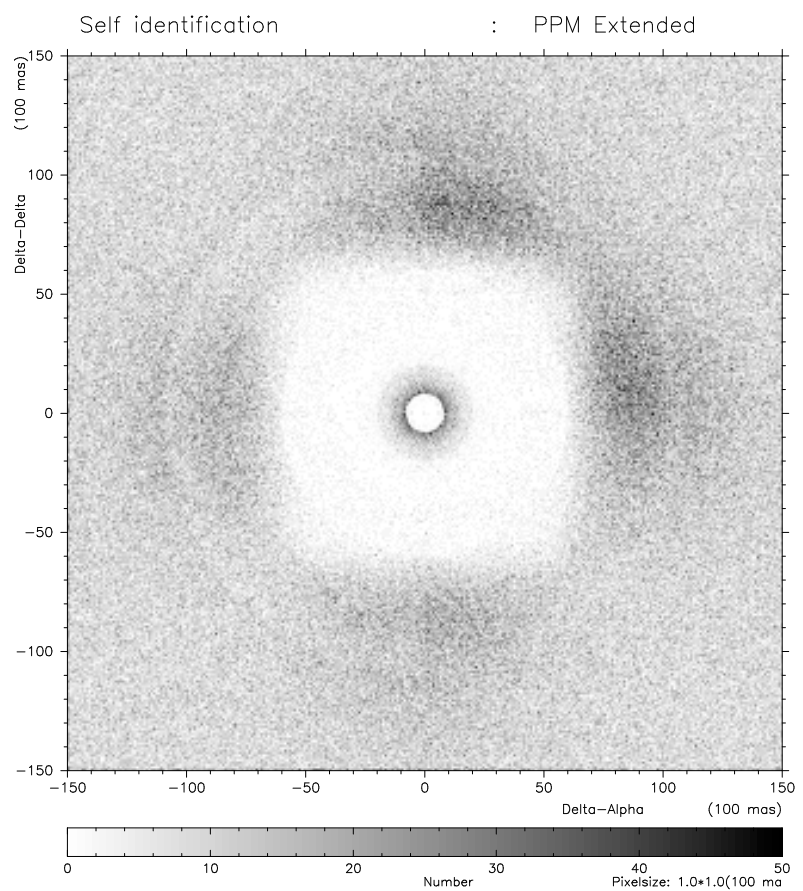

Fig. 5. The autocorrelation function of PPMX.

improvement. We also deleted fainter companions closer than 6 arcsec to a brighter star, if the fainter companion was only detected in GSC 1.2 as the earliest epoch. Figure 5 shows the 2dimensional autocorrelation function of PPMX. As can be seen, most of the spurious entries from GSC 1.2 and 2MASS are gone. There is a remaining faint asymmetry north-east vs. southwest of spurious stars whose origin could not be resolved. PPM and Tycho-2 have a better spatial resolution than GSC 1.2 or 2MASS, therefore we have kept double stars with flags "P" and "T" in Col. 27 even if their separations are less than 6 arcsec. The increase in density towards 1 arcsec separation originates from physical double stars contained in Tycho-2.

\subsection{Astrometry in PPMX}

The individual catalogues have been reduced to the Tycho-2 system. Thus, remaining systematic deviations of, e.g., the proper motion system of PPMX from that of Tycho-2 should not be expected. A comparison showed that they are smaller than $1 \mathrm{mas} /$ year on scales of a square degree or larger. There is, however, an elegant way to test the proper motion system of a star catalogue. The proper motions of a catalogue in an inertial reference system should only reflect the physical motions of the stars in our Galaxy, i.e. the reflex of solar motion and the rotation of the Galaxy. Systematics parallel to the axes of right ascension or declination must not appear, nor should one see features representing the plate lay-out of a photographic survey.

Figures 6 and 7 show the proper motions of the survey part of PPMX in the right ascension, declination plane. The major feature to be seen in these plots is the solar reflex motion. The size of the effect is such that it resembles a stellar sample with a typical distance of 400 to 500 parsec from the sun. There is an artificial depression of 1 to 2 mas/y in the proper motions in RA at declination +40 degrees, which is already inherent in Tycho- 2 . The reason is unknown; it only correlates with the transition

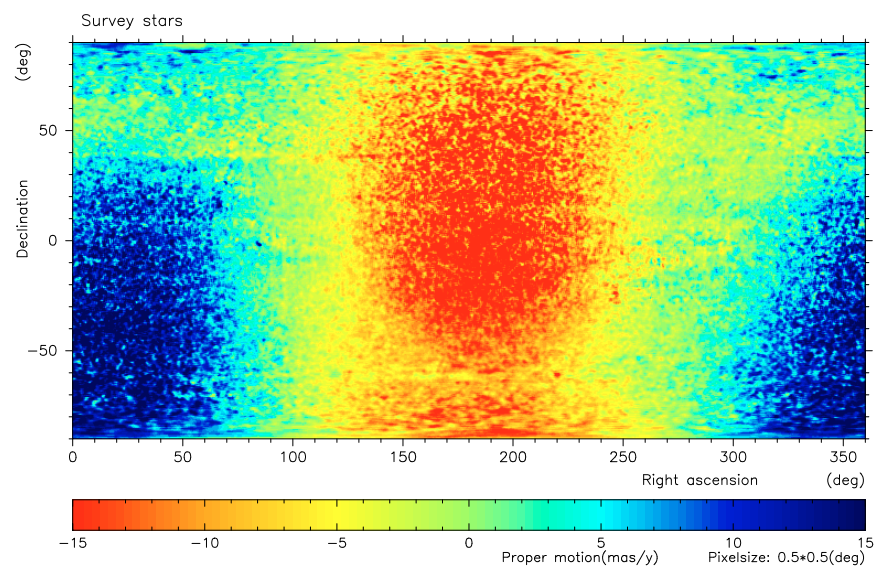

Fig. 6. The proper motions in right ascension averaged in 0.25 square degree bins plotted over right ascension and declination for stars in the survey. The major effect seen here is the reflex of solar motion. The amplitude of the effects tells us that the bulk of the survey stars has distances between about 400 and $500 \mathrm{pc}$ from the sun.

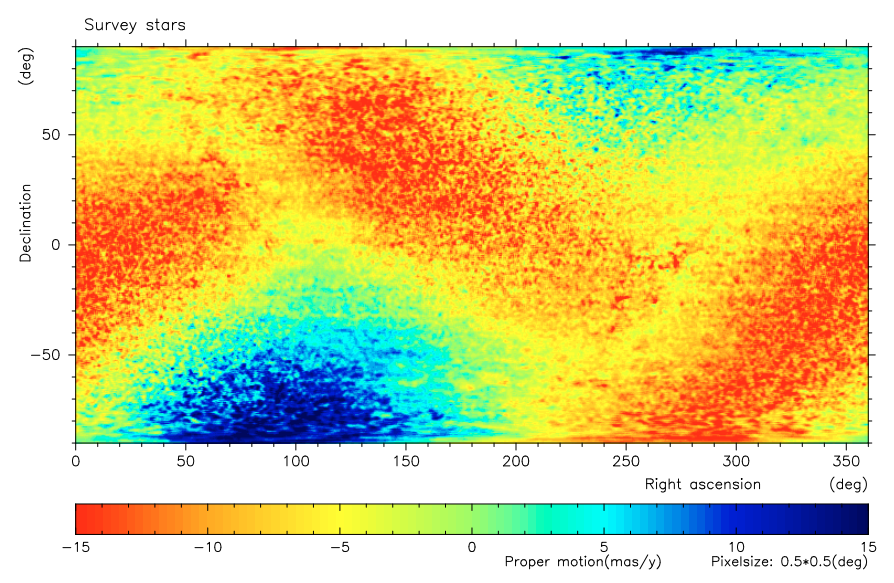

Fig. 7. The same as Fig. 6, but for the proper motions in declination.

from the Potsdam/Hyderabad zone of AC to the Helsingfors zone.

For non-survey stars which, at the same time, are not in the high-precision subset, the plate pattern of GSC 1.2 can be seen in proper motion plots such as Fig. 6 at a 2 mas/y level, especially in the northern declination range from the equator to +50 degrees. This plot is not shown in the paper. The effect resembles the regions of plate overlap. As in these overlap regions the number density of stars is larger, too, it is unclear if the origin of these systematics is a consequence of the higher number density or comes from still unresolved biases in the GSC 1.2 plate reductions. The user is adviced to consider this effect in applications based on stars with the "O" flag in Col. 27.

The mean errors of the PPMX proper motions are determined in the LSQ adjustment using Eq. (1) with an a priori error of unit weight arbitrarily set to 1 . Due to the generally small number of observations, hence low degree of freedom, an a posteriori determination of the error of unit weight from the residues per star leads to unreliable results. If, however, we used all residuals of all stars, we found that the a posteriori error of unit weight turned out to be about 5 percent smaller than the a priori one, which proves that the error assignment to the observational catalogues was correct. 

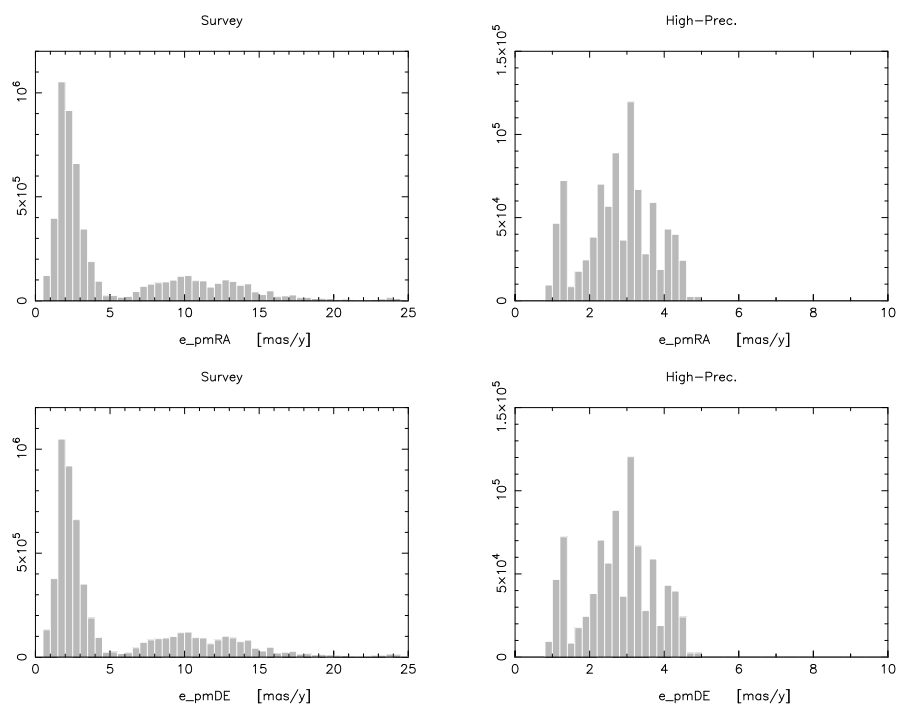

Fig. 8. Histogram of the mean errors of proper motions in PPMX. The left panels show the distribution for the stars in the survey, the right panels for the high precision subset of PPMX.

The distribution of the formal mean errors of the PPMX proper motions are shown in Fig. 8.

Here the distributions of the mean errors in proper motions of RA and Dec. are presented, both for the "survey" part (left panels) and the additional high-precision stars. Due to the selection criteria for the survey, the distribution is bi-modal . Two thirds of the stars in the survey have AC observations and their mean errors peak between 1.5 and 2 mas/y. The remaining stars have only observations in 2MASS/UCAC2/CMC14 at the modern epoch and GSC 1.2 at the old epoch and show a broad distribution between 5 and 15 mas/y. This mirrors the distribution of the GSC 1.2 epochs ranging from the mid-seventies to the mid-eighties of the last century. The distribution for the highprecision stars (right panels) shows many individual peaks. The reason for this is attributed to the Astrographic Catalog. The individual zones of the $\mathrm{AC}$ have different accuracies of measurements and, also, greatly vary in their epochs. Later epochs give smaller epoch differences and hence a poorer accuracy in the proper motions. As frequently mentioned, this subset of highprecision proper motions is NOT magnitude limited. All the remaining stars in the catalogue (flagged "O") show the same distribution as the low-precision part of the survey. Only a minority of the "O" stars have significant (more than 3 times their mean errors) proper motions, but all are published to create no selection effect.

\subsection{Photometry in PPMX}

Photometric information in PPMX is copied from the source catalogues. All stars are given a "magnitude" in Col. 12 of the catalogue. This magnitude is taken from the source catalogue of Col. 27. For the overwhelming majority these are GSC magnitudes. If the source is Tycho-2, the $V_{\mathrm{T}}$ magnitude is given. For $99.8 \%$ of the stars, photometry from 2MASS is available. For stars included in the ASCC 2.5, $B$ and $V$ magnitudes in the Johnson system are given (Cols. 14 and 16). To construct a magnitude limited survey we have determined an artificial $R_{J}$ magnitude in the UCAC2 photometric system from 2MASS
Table 1. Contents of the PPM-extended catalogue.

\begin{tabular}{|c|c|c|}
\hline Label & Units & Explanations \\
\hline 1 name & - & Name \\
\hline 2 RAmas & mas & Right Ascension J2000.0, epoch 2000.0 \\
\hline 3 DEmas & mas & Declination J2000.0, epoch 2000.0 \\
\hline 4 pmRA & $0.01 \mathrm{mas} / \mathrm{yr}$ & Proper Motion in RA $\cdot \cos (\mathrm{DE})$ \\
\hline $5 \mathrm{pmDE}$ & $0.01 \mathrm{mas} / \mathrm{yr}$ & Proper Motion in DE \\
\hline $6 T_{\mathrm{RA}}$ & $0.01 \mathrm{yr}$ & Mean Epoch (RA) - 2000.00 \\
\hline $7 T_{\mathrm{DE}}$ & $0.01 \mathrm{yr}$ & Mean Epoch (DE) - 2000.00 \\
\hline $8 e_{\mathrm{RA}}$ & mas & Mean error of Right Ascension \\
\hline $9 e_{\mathrm{DE}}$ & mas & Mean error of Declination \\
\hline $10 e_{\mathrm{pmRA}}$ & $0.1 \mathrm{mas} / \mathrm{yr}$ & Mean error of $\mathrm{pmRA} \cdot \cos (\mathrm{DE})$ \\
\hline $11 e_{\mathrm{pmDE}}$ & $0.1 \mathrm{mas} / \mathrm{yr}$ & Mean error of pmDE \\
\hline $12 G$ & $0.001 \mathrm{mag}$ & $\begin{array}{l}G \text { magnitude from source catalogue } \\
\text { (column 27) }\end{array}$ \\
\hline $13 R_{J}$ & $0.001 \mathrm{mag}$ & calculated $R_{J}$ magnitude (see text) \\
\hline $14 B$ & $0.001 \mathrm{mag}$ & Johnson $B$ magnitude from ASCC-2.5 \\
\hline $15 e_{B}$ & $0.001 \mathrm{mag}$ & Standard error of $B$ magnitude \\
\hline $16 \mathrm{~V}$ & $0.001 \mathrm{mag}$ & Johnson $V$ magnitude from ASCC-2.5 \\
\hline $17 e_{V}$ & $0.001 \mathrm{mag}$ & Standard error of $V$ magnitude \\
\hline $18 \mathrm{~J}$ & $0.001 \mathrm{mag}$ & $J$ magnitude from 2 MASS \\
\hline $19 e_{J}$ & $0.001 \mathrm{mag}$ & Standard error of $J$ magnitude \\
\hline $20 \mathrm{H}$ & $0.001 \mathrm{mag}$ & $H$ magnitude from 2MASS \\
\hline $21 e_{H}$ & $0.001 \mathrm{mag}$ & Standard error of $H$ magnitude \\
\hline $22 K$ & $0.001 \mathrm{mag}$ & $K$ magnitude from 2 MASS \\
\hline $23 e_{K}$ & $0.001 \mathrm{mag}$ & Standard error of $K$ magnitude \\
\hline $24 n_{\text {obs }}$ & - & number of observations in the LSQ solution \\
\hline $25 \mathrm{P}$ & - & P flag. Bad LSQ fit \\
\hline \multirow[t]{3}{*}{$26 \mathrm{sub}$} & - & Subset flag: \\
\hline & & "survey $(\mathrm{S})$ ", \\
\hline & & $\begin{array}{l}\text { "additional high-precision stars }(\mathrm{H}) \text { " or } \\
\text { "additional stars (O)" }\end{array}$ \\
\hline \multirow[t]{7}{*}{$27 I_{\mathrm{sc}}$} & - & Index of source catalogue \\
\hline & & H stars from ARIHIP (Wielen et al.) \\
\hline & & T stars from Tycho-2 (Hoeg et al.) \\
\hline & & P stars from PPM (Röser and Bastian 1991) \\
\hline & & S stars from STARNET (Röser 1996) \\
\hline & & G stars (matching GSC 1.2 with 2MASS) \\
\hline & & A stars (matching AC2000.2 with 2MASS) \\
\hline $28 \mathrm{Id}$ & - & Identifier in the source catalogue \\
\hline
\end{tabular}

photometry according to the method developed in Piskunov et al. (2008) (see Sect. 4.1). This magnitude is given in Col. 13.

\section{The catalogue}

The catalogue is subdivided into 24 declination zones of $7.5 \mathrm{deg}$ each. Stars in the zones are ordered by increasing right ascension. The contents of the catalogue are described in Table 1. The first column gives the name of the star (in IAU convention HHMMSS.S \pm DDMMSS). The prefix "PPMX J" should be added to identify a star in the catalogue. There are 346 cases in which different stars would be attributed identical names. Therefore, suffixes $\mathrm{p}$ (preceding) or $\mathrm{f}$ (following) are appended to distinguish between them. Then follows the astrometric information (Cols. 2 to 11), right ascension and declination at equinox and epoch 2000.0 in the ICRS system and the respective proper motions, as well as the full covariance matrix of these quantities. This includes the mean epoch, mean error of the coordinates at the mean epoch and mean errors of proper motions. Columns 12 to 22 contain the photometric information as described in Sect. 4.4. Columns 24 and 25 present the number of observations used in the weighted LSQ solution, and a P flag if the $\chi^{2}$ of the fit was unacceptable. There are 229618 (or 1.6\%) 
cases with P flags in the catalogue. Column 24 is left "blank", if the stars originate from ARIHIP (" $\mathrm{H}$ " in Col. 27) or from Tycho-2 ("T" in Col. 27). Column 26 describes if a star belongs to the survey " $\mathrm{S}$ ", to high precision proper motion stars "H" fainter than the survey limit, or the remaining stars as "O" (see Sect. 4.1). Column 27 gives the source catalogues for the star list (see Sect. 3.1), and Col. 28 is the name of the star in the source catalogue.

This catalogue will be made available via the CDS, Strasbourg, France.

\subsection{Caveats}

Firstly, stars with high proper motions (HPM), having a total proper motion value larger than about $200 \mathrm{mas} / \mathrm{y}$ and only 2 or 3 observations (Col. 24 in the catalogue) may be spurious. Their proper motions are effectively based on two epochs only, GSC1.2 as the first and 2MASS/UCAC2/CMC14 as the second. If one is interested in a particular star, it is advisable to crossmatch with USNO-A2.0, especially in the northern hemisphere. Cross-matching of all the HPM stars was beyond the scope of the present paper; it will be done in the near future. There are some 39000 stars with proper motions higher than 200 mas/y, but 16000 of them carry a "P" (problem) flag in Col. 25.

Secondly, if a star in the catalogue has its nearest neighbour closer than 10 arcsec, the fainter one of this pair may be spurious, as seen in Fig. 5. The pattern in Fig. 5 is a result of the spurious entries in Figs. 1 and 2 from GSC 1.2, and 3 from 2MASS. The observed overdensity in Fig. 5 gives rise to about 19000 of these cases.

Doubtful cases have been retained in PPMX. We publish them because, to a certain degree, they are measurements (or "observations"). In order to verify in the future what has been done, these "observations" must be made available.

Acknowledgements. Part of this work was supported by DFG grant 436 RUS $113 / 757 / 0-2$, and RFBR grants 06-02-16379 and 07-02-91566. This paper is based on observations from the ESA Hipparcos satellite. This publication makes use of data products from the Two Micron All Sky Survey, which is a joint project of the University of Massachusetts and the Infrared Processing and Analysis Center/California Institute of Technology, funded by the National Aeronautics and Space Administration and the National Science Foundation. This research has made use of the SIMBAD database, operated at CDS, Strasbourg, France

\section{References}

Bystrov, N. F., Polojentsev, D. D., \& Potter, H. I. 1994, Bull. Inf. CDS, 44, 3 (1994)

Copenhagen Univ. Obs., Inst. of Astronomy, Cambridge, UK, \& Real Instituto Y Observatorio de La Armada, F. E. S. 2006, VizieR Online Data Catalog, 1304,0

ESA 1997, VizieR Online Data Catalog, 1239, 0

Høg, E., Fabricius, C., Makarov, V. V., et al. 2000a, A\&A, 357, 367

Høg, E., Fabricius, C., Makarov, V. V., et al. 2000b, A\&A, 355, L27

Kharchenko, N. V. 2001, Kinematika i Fizika Nebesnykh Tel, 17, 409

Kopff, A., Nowacki, H., \& Strobel, W. 1964, Veröff. Astron. Rechen-Inst. Heidelberg No. 14

Lasker, B. M., Sturch, C. R., McLean, B. J., et al. 1990, AJ, 99, 2019

Monet, D. G., Levine, S. E., Canzian, B., et al. 2003, AJ, 125, 984

Morrison, J. E., Röser, S., McLean, B., Bucciarelli, B., \& Lasker, B. 2001, AJ, 121,1752

Piskunov, A. E., Kharchenko, N. V., \& Chupina, N. V. 2008, Astron. Lett., 34, 256.

Rapaport, M., Ducourant, C., Le Campion, J. F., et al. 2006, A\&A, 449, 435

Röser, S. 1996, IAU Symp., 172, 481

Röser, S., \& Bastian, U. 1991, PPM Star Catalogue, Heidelberg (New York: Published for Astronomisches Rechen-Institut by Spektrum Akademischer Verlag)

Röser, S., \& Høg, E. 1993, TYCHO Reference Catalogue (TRC) Workshop on Databases for Galactic Structure (1993) ed. A. G. D. Philip, B. Hauck, A. R. Upgren, \& L. Davis, 137

Skrutskie, M. F., Cutri, R. M., Stiening, R., et al. 2006, AJ, 131, 1163

Urban, S. E., Corbin, T. E., Wycoff, G. L., et al. 1998, AJ, 115, 1212

Urban, S. E., Corbin, T. E., Wycoff, G. L., et al. 2001, BAAS, 33, 1494

van Leeuwen, F. 2007, Hipparcos, the New Reduction of the Raw Data. By Floor van Leeuwen, Institute of Astronomy, Cambridge University, Cambridge (UK Series: Astrophysics and Space Science Library, Dordrecht: Springer), 350, 20

Wielen, R., Lenhardt, H., Schwan, H., \& Dettbarn, C. 1999, A\&A, 347, 1046

Wielen, R., Schwan, H., Dettbarn, C., et al. 2001, Veroeffentlichungen des Astronomischen Rechen-Instituts Heidelberg, 40, 1

Zacharias, N., Urban, S. E., Zacharias, M. I., et al. 2004, AJ, 127, 3043 